\title{
5 Analytic Continuation
}

\subsection{Analytic Continuation}

Our focus in the last chapter was on the construction of an analytic function from a knowledge of its singularities. More often than not, however, we are confronted with the inverse problem: given some knowledge of a function in a restricted region of its domain of holomorphy, determine its singularities. This will be the focus of the present chapter.

We have seen repeatedly that one need not know all that much about an analytic function in order to determine its value everywhere in the complex plane or on its Riemann surface. Cauchy's Integral Representation can be viewed as the embodiment of this property and thus far it has provided the key to exploiting it. We are now going to find out what constitutes a minimal set of information for the determination of an analytic function. The answer is one that is best exploited not by Cauchy's Integral but by one of its consequences, the Taylor series. In so doing, we shall also find out how to use a representation of a function that is valid in one domain of the complex plane to determine its values at points outside the domain or indeed, at any points where it is holomorphic.

Our starting point is the following theorem which, despite its innocuous appearance, is one of the most remarkable results of complex analysis.

Theorem: Let $f_{1}(z)$ and $f_{2}(z)$ be holomorphic in a domain $D$ of the complex plane. If the two functions coincide in any neighbourhood, however small, of a point $z$ in $D$, or even on a point set with an accumulation point in $D$, then they coincide throughout D.

Proof: The function $f_{1}(z)-f_{2}(z)$ is holomorphic throughout $D$ and has a set of zeros consisting of the points where $f_{1}(z)$ and $f_{2}(z)$ coincide, with an accumulation point in $D$. We know that in any domain where it is holomorphic a function either has isolated zeros or it is identically zero. Thus, $f_{1}(z)-f_{2}(z) \equiv 0$ or, $f_{1}(z) \equiv f_{2}(z)$, for all $z$ in $D$.

What this theorem establishes is that a holomorphic function is uniquely determined everywhere within its domain of holomorphy by its behaviour in the neighbourhood of an arbitrary point of that domain. But how can one exploit this remarkable property? Obviously not by means of a Cauchy Integral or dispersion representation or anything else of that ilk as we lack the necessary input information. However, what we do have is precisely the information needed to determine a Taylor series representation.

Suppose that we know the value of the function $f(z)$ throughout a neighbourhood of the point $z=z_{0}$ which is a point lying within the function's domain of holomorphy, $D$. This is sufficient to permit calculation of the coefficients

$$
c_{0}=f\left(z_{0}\right), c_{1}=f^{\prime}\left(z_{0}\right), \ldots, c_{m}=\frac{1}{m !} f^{(m)}\left(z_{0}\right), \ldots
$$


of the Taylor expansion of $f(z)$ about $z=z_{0}$. Hence, we can set

$$
f(z)=\sum_{m=0}^{\infty} c_{m}\left(z-z_{0}\right)^{m},
$$

for all values of $z$ within a circle $C_{0},\left|z-z_{0}\right|=R_{0}$. The quantity $R_{0}$, the radius of convergence of the series, is equal to the distance from $z_{0}$ to the nearest singularity of $f(z)$ and so will normally be larger than the radius of the neighbourhood from which we started. Thus, equation (5.1.1) provides us with an analytic continuation of $f(z)$ which means that it determines the behaviour of $f(z)$ outside its initial domain of definition. Moreover, our theorem guarantees that this analytic continuation is unique.

The disc in which (5.1.1) converges likely represents a small fraction of the domain of holomorphy $D$ and so may not include a particular point $z=z_{0}^{\prime}$ that is of interest to us. To analytically continue $f(z)$ to such a distant point requires either a different representation, with a much larger domain of validity, or a process involving the repeated generation of Taylor series with overlapping circles of convergence. We shall consider the second option first.

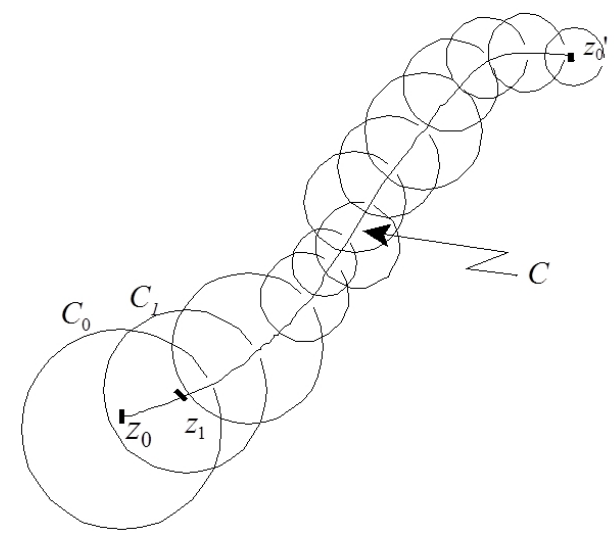

Figure 5.1: A function can be analytically continued along a curve $C$ by means of repeated Taylor expansions about appropriately chosen points of $C$.

From the definition of a domain we know that the points $z_{0}$ and $z_{0}^{\prime}$ can be connected by a simple curve $C$ that lies entirely within $D$. As shown in Figure 5.1, let us take a point $z_{1}$ on $C$ such that $\left|z_{1}-z_{0}\right|<R_{0}$, that is, such that $z_{1}$ lies within the circle of convergence $C_{0}$ of the Taylor series in (5.1.1).

Since the series converges uniformly in every closed disc $\left|z-z_{0}\right| \leq r<R_{0}$, it can be differentiated term by term to determine all derivatives of $f(z)$ at all points of such a disc. In particular, since we may choose an $r$ for which $\left|z_{1}-z_{0}\right|<r<R_{0}$, we can 
calculate

$$
\begin{aligned}
f\left(z_{1}\right) & =\sum_{m=0}^{\infty} c_{m}\left(z_{1}-z_{0}\right)^{m}, f^{\prime}\left(z_{1}\right)=\sum_{m=1}^{\infty} c_{m} m\left(z_{1}-z_{0}\right)^{m-1}, \ldots, \\
\frac{1}{n !} f^{(n)}\left(z_{1}\right) & =\sum_{m=n}^{\infty} c_{m} \frac{m !}{n !(m-n) !}\left(z_{1}-z_{0}\right)^{m-n}, \ldots
\end{aligned}
$$

But these are just the coefficients $c_{m}^{(1)}, m=0,1, \ldots, n, \ldots$ of the Taylor series expansion of $f(z)$ about the point $z=z_{1}$. Hence, we can now set

$$
f(z)=\sum_{m=0}^{\infty} c_{m}^{(1)}\left(z-z_{1}\right)^{m}
$$

which is valid for all $z$ within a circle $C_{1}$ with centre at $z=z_{1}$ and radius $R_{1}$.

Since $f(z)$ is holomorphic at all points on $C, R_{1}$ must be non-zero and hence, it must be possible to choose the point $z=z_{1}$ so that $C_{1}$ lies partly outside $C_{0}$. Therefore, (5.1.3) provides a unique analytic continuation of $f(z)$ from $C_{0}$ to the somewhat larger domain formed by the union of $C_{0}$ and $C_{1}$. On the segment of the curve $C$ that lies outside $C_{0}$ but within $C_{1}$ we now choose a new point $z=z_{2}$. Then, using the uniformly convergent series in (5.1.3) to calculate the coefficients of the Taylor expansion of $f(z)$ about $z=z_{2}$, we obtain a further analytic continuation of $f(z)$. Repeating this argument over and over, we proceed along $C$ with overlapping circles $C_{0}, C_{1}, C_{2}, \ldots$ until one of the circles finally covers the point $z=z_{0}^{\prime}$ thus enabling us to find the Taylor expansion of $f(z)$ about it as well. The behaviour of $f(z)$ in the neighbourhood of $z=z_{0}^{\prime}$ is then determined and our analytic continuation procedure is completed.

It is not possible to analytically continue through a singular point of $f(z)$ since the radii of the circles $C_{i}$ tend to zero as we approach it. However, it is possible to analytically continue around the singularity and in the process, determine its location; (see Figure 5.2). Thus, in principle at least, this technique can be used to continue a function throughout its domain of holomorphy, starting from an arbitrary point of that domain and using all possible paths to form chains of overlapping circles. The continuation will be complete when the domain's natural boundaries, which are just the singular points of the function, have all been encountered. This also applies to mutivalued functions for if we analytically continue around a branch point on one Riemann sheet, we will eventually generate values appropriate to an adjacent sheet. Thus, continuing along all possible paths we will determine the behaviour of the function throughout its Riemann surface as well as the geometry of that surface.

As we have emphasized above, the only barrier this technique cannot surmount is the natural boundary of the function's domain of holomorphy. There are some functions for which this is surprisingly limiting, at least in a geometrical sense. Consider for example,

$$
f(z)=1+z^{2}+z^{4}+z^{8}+z^{16}+\ldots=1+\sum_{m=1}^{\infty} z^{2^{m}},
$$




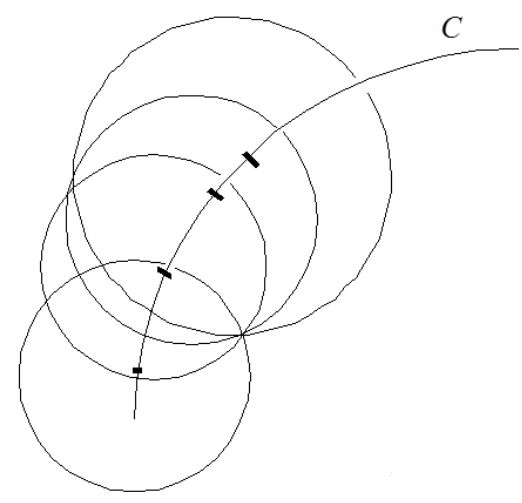

Figure 5.2: Analytic continuation around a singular point.

whose circle of convergence is $|z|=1$. It is readily shown that any value of $z$ that satisfies one of the equations

$$
z^{2}=1, z^{4}=1, z^{8}=1, \ldots, z^{2^{m}}=1, \ldots
$$

is a singularity of $f(z)$. These values correspond to the points $z=e^{2 \pi i k / 2^{m}}$, where $k$ and $m$ are integers. On every arc of the unit circle there is an infinite number of such points. Thus, it is impossible to continue $f(z)$ outside its circle of convergence since it is also the natural boundary of the function's domain of holomorphy.

While conceptually powerful, the technique of successive Taylor expansion of a function is impractical. Fortunately, there are many alternative and more immediate methods to effect analytic continuations. Before we examine some of them, however, we need to delve a little deeper into the implications of the theorem that introduced this Section.

Suppose that we are given two analytic functions $f_{1}(z)$ and $f_{2}(z)$ whose functional forms differ from each other and are valid only in the domains $D_{1}$ and $D_{2}$, respectively. Suppose further that $D_{1}$ and $D_{2}$ overlap and that in their intersection $f_{1}(z)$ and $f_{2}(z)$ are identical. Then, our theorem tells us that the (unique) result of analytically continuing $f_{1}(z)$ into $D_{2}$ must coincide with $f_{2}(z)$ and conversely the result of analytic continuation of $f_{2}(z)$ into $D_{1}$ must be identical to $f_{1}(z)$. In fact, $f_{1}(z)$ and $f_{2}(z)$ are just local representations of the unique function

$$
f(z)=\left\{\begin{array}{l}
f_{1}(z), z \text { in } D_{1} \\
f_{2}(z), z \text { in } D_{2}
\end{array}\right.
$$

which is holomorphic throughout the combined domain $D_{1} \cup D_{2}$.

Since the application of analytic continuation to $f_{1}(z)$ yields $f_{2}(z)$ and vice versa, one says that $f_{1}(z)$ and $f_{2}(z)$ are analytic continuations of each other. 
Example: Consider the functions

$$
f_{1}(z)=\sum_{m=0}^{\infty} \frac{z^{m}}{a^{m+1}}, \quad|z|<|a|
$$

and

$$
f_{2}(z)=\sum_{m=0}^{\infty} \frac{(z-b)^{m}}{(a-b)^{m+1}}, \quad|z-b|<|a-b|
$$

where, to ensure that the circle of convergence of $f_{2}(z)$ is not interior to that of $f_{1}(z)$, we require that $b / a$ not be real. Both series sum to the function $(a-z)^{-1}$. Thus, although $f_{1}(z)$ and $f_{2}(z)$ have different functional forms defined in different albeit overlapping domains, they represent the same analytic function, $f(z)=\frac{1}{a-z}$. Each is a unique analytic continuation of the other but, in particular, $f_{2}(z)$ provides the means of analytically continuing $f_{1}(z)$ to any point outside the circle $|z|=|a|$ simply by varying the value of the parameter $b$. However, if this is our primary interest we are by no means restricted to the use of power series representations to accomplish it. Indeed, the two integral representations

$$
\begin{aligned}
& f_{3}(z)=\int_{0}^{\infty} e^{-t(a-z)} d t, \quad \operatorname{Re} z<\operatorname{Re} a \\
& f_{4}(z)=-\int_{0}^{\infty} e^{t(a-z)} d t, \quad \operatorname{Re} z>\operatorname{Re} a
\end{aligned}
$$

which converge to $(a-z)^{-1}$ in their respective domains of definition, provide a more effective means of analytically continuing $f_{1}(z)$ outside its circle of convergence.

In this example we have a closed-form expression for $f(z)$, namely $(a-z)^{-1}$, which is valid throughout the function's domain of holomorphy. However, this is an exception rather than a norm. The functions that can be expressed in terms of a finite number of elementary functions make up a very limited subset of the totality of analytic functions. We must get used to the idea that to know how a function behaves at widely separated points one usually requires two or more representations of very diverse appearance. A perfect example is provided by a function called the gamma function and denoted $\Gamma(z)$. Before introducing it, however, we need to complete our discussion of analytic continuation.

We have required that two functions $f_{1}(z)$ and $f_{2}(z)$ have overlapping domains of definition if they are to be analytic continuations of each other. As our next theorem shows, this is unnecessarily restrictive.

Theorem: Let $f_{1}(z)$ and $f_{2}(z)$ be holomorphic in the simply connected domains $D_{1}$ and $D_{2}$, respectively and let $D_{1}$ and $D_{2}$ have in common as part of their boundaries a simple curve $C$. Then, $f_{1}(z)$ and $f_{2}(z)$ are analytic continuations of each other if and 


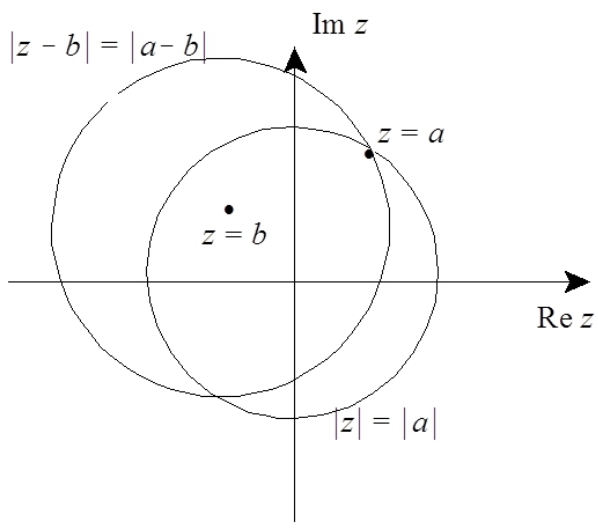

Figure 5.3: The domains of definition of the two representations of $f(z)=(a-z)^{-1}$ given in equations (5.1.4) and (5.1.5).

only if they tend uniformly to common values along $C$; that is, if and only if they are continuous in the regions $D_{1} \cup C$ and $D_{2} \cup C$, respectively and $f_{1}(z)=f_{2}(z)$ for all $z$ on C.

Proof: That the condition is necessary is obvious. Therefore, we need only show that it is sufficient.

Define the function $f(z)$ as follows:

$$
f(z)=\left\{\begin{array}{l}
f_{1}(z) \text { for } z \text { in } D_{1} \text { or } C \\
f_{2}(z) \text { for } z \text { in } D_{2} \text { or } C .
\end{array}\right.
$$

We must now show that $f(z)$ is holomorphic throughout the entire domain

$$
D=D_{1} \cup D_{2} \cup C
$$

Let $\Gamma$ be any simple closed curve within $D_{1} \cup D_{2} \cup C$ and consider the integral

$$
\int_{\Gamma} f(z) d z
$$

If $\Gamma$ lies entirely within either $D_{1}$ or $D_{2}$, the integral vanishes by Cauchy's Theorem. If $\Gamma$ lies in both $D_{1}$ and $D_{2}$ then, as shown in Figure 5.4, we can introduce two simple closed curves $\Gamma_{1}$ and $\Gamma_{2}$ separated by an infinitesimal distance so that they lie entirely within $D_{1}$ and $D_{2}$, respectively and follow a section of the boundary $C$ in opposite directions. We can thereby write (5.1.6) as the sum of two integrals,

$$
\int_{\Gamma} f(z) d z=\int_{\Gamma_{1}} f(z) d z+\int_{\Gamma_{2}} f(z) d z=\int_{\Gamma_{1}} f_{1}(z) d z+\int_{\Gamma_{2}} f_{2}(z) d z,
$$



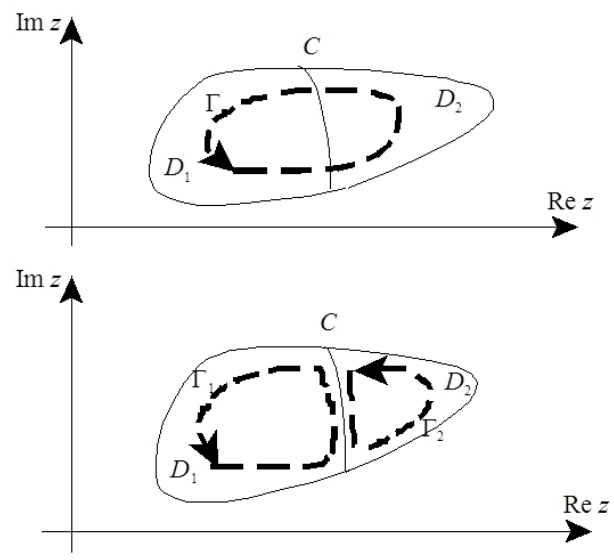

Figure 5.4: A simple closed curve $\Gamma \in D_{1} \cup D_{2} \cup C$ is separated at the boundary $C$ into two simple closed curves $\Gamma_{1}$ and $\Gamma_{2}$.

both of which vanish. Thus,

$$
\int_{\Gamma} f(z) d z=0
$$

for any closed contour $\Gamma$ contained within the simply connected domain $D_{1} \cup D_{2} \cup C$ and so, by Morera's Theorem, $f(z)$ is holomorphic there. Therefore, $f_{1}(z)$ and $f_{2}(z)$ are analytic continuations of each other.

We are now in a position to prove a theorem which not only generalizes a common feature of the elementary functions but provides an analytic continuation technique that plays a key role in most physical applications of dispersion representations. It is known as the Schwarz reflection principle.

Theorem: Let $f(z)$ be holomorphic in a domain $D$ which has, as part of its boundary, a segment $C$ of the real axis and let $D^{*}$ be the mirror image of $D$ with respect to that axis. Then, if $f(z)$ is continuous within the region $D \cup C$ and assumes real values on $C$, its analytic continuation into the domain $D^{*}$ exists and is given by $f^{\star}\left(z^{\star}\right)$ for all $z$ in $D^{*}$.

Proof: Let $\Gamma$ denote an arbitrary simple closed curve in $D$, described by the parametric equation $z=\zeta(t), t_{1} \leq t \leq t_{2}$. Since $f(z)$ is holomorphic in $D$, we have

$$
\int_{\Gamma} f(z) d z=\int_{t_{1}}^{t_{2}} f[\zeta(t)] \frac{d \zeta(t)}{d t} d t=0 .
$$

Let $\Gamma^{\star}$ be the image of $\Gamma$ in $D^{\star}$, (Figure 5.5). Its parametric equation must then be $z=$ $\zeta^{\star}(t), t_{1} \leq t \leq t_{2}$, with increasing $t$ corresponding to a clockwise (counter-clockwise) traversal of $\Gamma^{\star}$ if it produces a counter-clockwise (clockwise) circuit of $\Gamma$. Thus, inte- 
grating the function $g(z) \equiv f^{\star}\left(z^{\star}\right)$ around $\Gamma^{\star}$, we have

$$
\int_{\Gamma^{\star}} g(z) d z=\int_{t_{1}}^{t_{2}} g\left[\zeta^{\star}(t)\right] \frac{d \zeta^{\star}(t)}{d t} d t=\int_{t_{1}}^{t_{2}} f^{\star}[\zeta(t)] \frac{d \zeta^{\star}(t)}{d t} d t=\left[\int_{t_{1}}^{t_{2}} f[\zeta(t)] \frac{d \zeta(t)}{d t} d t\right]^{\star}=0
$$

by equation (5.1.7) . Hence, we know from Morera's Theorem that $f^{\star}\left(z^{\star}\right)$ is a holomorphic function of $z$ throughout the simply connected domain $D^{\star}$. Moreover, since $f(z)$ is real on the real axis segment $C$, we have $f^{\star}\left(z^{\star}\right)=f(z)$ for all $z$ on $C$. Therefore, according to the preceding theorem, $f(z)$ and $f^{\star}\left(z^{\star}\right)$ are analytic continuations of each other and together define the unique function

$$
F(z)=\left\{\begin{array}{c}
f(z), z \text { in } D \text { or } C \\
f^{\star}\left(z^{\star}\right), z \text { in } D^{\star} \text { or } C
\end{array}\right.
$$

which is holomorphic throughout the domain $D \cup D^{\star} \cup C$.

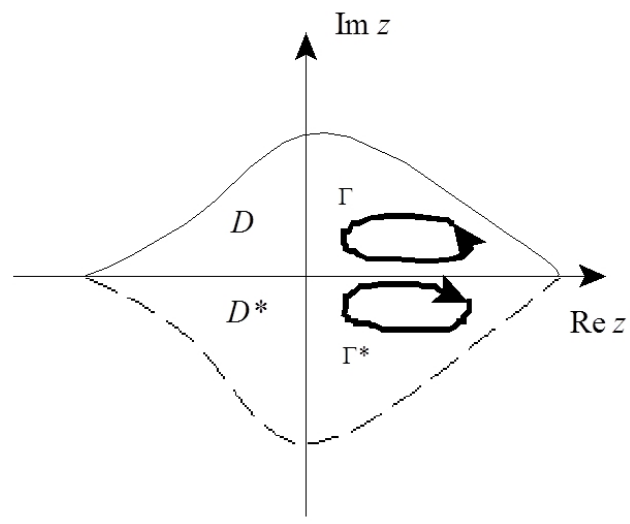

Figure 5.5: The domain $D$ and its mirror image through the real axis $D^{\star}$.

It follows immediately from (5.1.8) that

$$
F\left(z^{\star}\right)=F^{\star}(z) \text { for all } z \text { in } D \cup D^{\star} \cup C .
$$

Evidently, this relation must hold for any function that is holomorphic throughout a domain intersected by the real axis and that assumes real values when its argument is real. Thus, we now see why it was referred to as a reality condition in the preceding Chapter. More importantly, we also see that our discovery in Chapter 1 that it is satisfied by each of the single valued elementary functions was indicative of a general consequence of their definition and not mere coincidence. 


\subsection{The Gamma Function}

In this Section we shall introduce a variety of functional forms attributable to the gamma function which is one of the more frequently encountered functions of mathematical physics. This will illustrate just how small need be our reliance on Taylor series representations to effect analytic continuations and offer further exposure to a principal alternative, the use of integral representations.

The usual introduction to the gamma function is via Euler's integral (of the second kind) which, for real $x>0$, gives

$$
\Gamma(x)=\int_{0}^{\infty} e^{-t} t^{x-1} d t .
$$

Whether the introduction is made in a physics or a calculus course, it will always be pointed out that $\Gamma(x)$ is the continuous variable generalization of the factorial function, a fact that we can readily verify from equation (5.2.1). Writing $t^{x-1}$ as $\frac{1}{x} \frac{d}{d t} t^{x}$ and integrating by parts, we obtain

$$
\Gamma(x)=\left.\frac{e^{-t}}{x}\right|_{0} ^{\infty}+\int_{0}^{\infty} \frac{e^{-t}}{x} t^{x} d t
$$

The first term vanishes and so, using (5.2.1) to identify the second term, we have

$$
\Gamma(x+1)=x \Gamma(x), x>0 .
$$

Thus, since

$$
\Gamma(1)=\int_{0}^{\infty} e^{-t} d t=1,
$$

setting $x$ equal to $n$, an integer, yields

$$
\Gamma(n+1)=n !
$$

This makes it clear that $\Gamma(x)$ provides a smooth interpolation between the points defined by $n !, n=0,1,2, \ldots$; explicit evaluation yields the curve shown in Figure 5.6.

The integral in (5.2.1) continues to converge when $x$ is replaced by the complex variable $\mathrm{z}$ provided that $\operatorname{Re} z>0$. Moreover, differentiating the function

$$
\Gamma(z)=\int_{0}^{\infty} e^{-t} t^{z-1} d t, \quad \operatorname{Re} z>0
$$

with respect to $z$, we obtain yet another integral that converges for $\operatorname{Re} z>0$. Thus, (5.2.5) evidently defines an analytic function whose domain of holomorphy includes 
the right half-plane and hence, the positive real axis. Therefore, according to the theorem at the beginning of this Chapter, the integral in (5.2.5) is a representation for $\operatorname{Re} z>0$ of the only analytic function that can assume the values given by (5.2.1) when $\operatorname{Im} z=0$. Appropriately, we have denoted this function by $\Gamma(z)$. An alternative and somewhat simpler way of stating this result is to say that (5.2.5) is the analytic continuation of (5.2.1) from the positive real axis to the entire right half- plane.

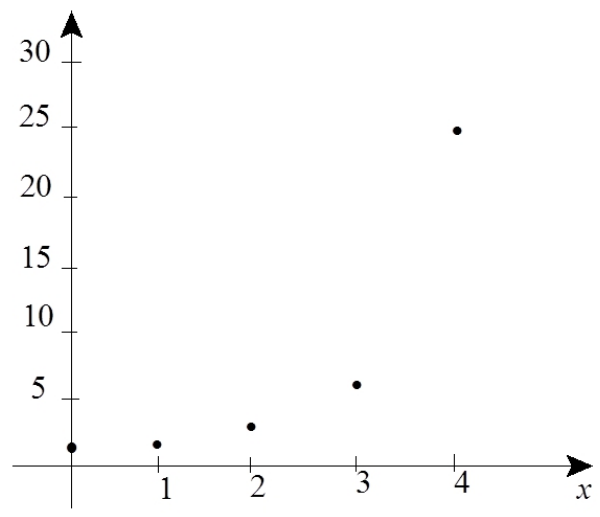

Figure 5.6: The dots indicate the discrete points defined by $n !$; the corresponding curve is $\Gamma(x+1)$.

With the help of the factorial property (5.2.2) we can use (5.2.5) to determine the natural boundary of the gamma function's domain of holomorphy. This will determine whether we need to seek an analytic continuation for $\operatorname{Re} z<0$ and if so, assist us with the search.

The factorial property holds for complex as well as real values of the arguments. In addition, we know that the integral representation of $\Gamma(z+1)$ converges for $\operatorname{Re} z>-1$ and hence, that the right hand side of

$$
z \Gamma(z)=\Gamma(z+1)
$$

is well-defined there. Thus, dividing through by $z$, we immediately obtain an analytic continuation of (5.2.5) from the domain $\operatorname{Re} z>0$ to $\operatorname{Re} z>-1$,

$$
\Gamma(z)=\frac{1}{z} \Gamma(z+1)=\frac{1}{z} \int_{0}^{\infty} e^{-t} t^{z} d t, \quad z \neq 0 .
$$

Evidently, $\operatorname{Re} z=0$ is not a natural boundary of $\Gamma(z)$. The sole source of the restriction $\operatorname{Re} z>0$ that has been placed on the integral representation (5.2.5) is an isolated singularity at $z=0$. Taking the limit of (5.2.7) as $z \rightarrow 0$ and recalling that $\Gamma(1)=1$, we 
find

$$
\Gamma(z) \rightarrow \frac{1}{z}
$$

which identifies the singularity as a simple pole and the corresponding residue as unity.

This procedure may be repeated as often as one likes to obtain

$$
\Gamma(z)=\frac{\Gamma(z+n+1)}{(z+n)(z+n-1)(z+n-2) \ldots(z)}
$$

the right hand of which is holomorphic for $\operatorname{Re} z>-n$ except for simple poles at the points $z=0,-1,-2, \ldots,-(n-1)$. Thus, we conclude that $\Gamma(z)$ is a meromorphic function and as such, can be continued anywhere in the left half-plane so long as we avoid its simple poles at the negative integers and zero. This allows, for example, calculation of its functional dependence on negative as well as positive real values of its argument as shown for $|x|<5$ in Figure 5.7 .

To find the residue of $\Gamma(z)$ at $z=-n$, we simply take the limit of (5.2.8) as $z \rightarrow-n$ and use $\Gamma(1)=1$. Thus,

$$
\Gamma(z) \rightarrow \frac{1}{(-1)^{n} n !(z+n)}
$$

and hence,

$$
\operatorname{Res}[\Gamma(-n)]=\frac{(-1)^{n}}{n !}
$$

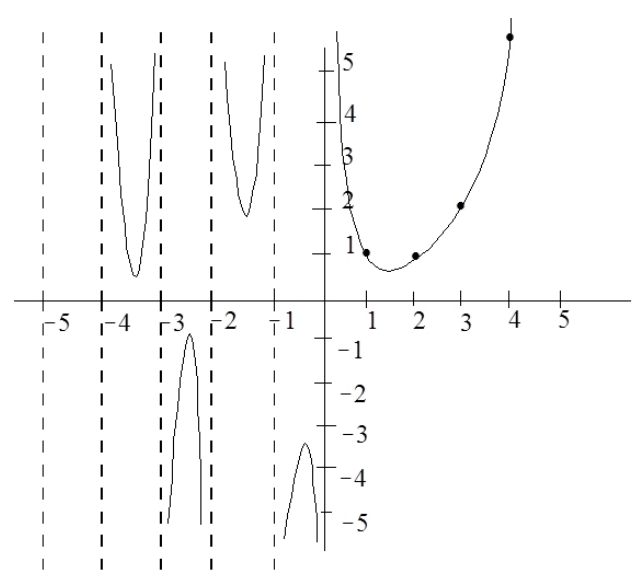

Figure 5.7: $\Gamma(z)$, as determined by (5.2.5) and (5.2.8), plotted for $z=x$, real.

An additional means of analytically continuing $\Gamma(z)$ into the left half-plane is provided by the product $\Gamma(z) \Gamma(1-z)$. The integral representation of $\Gamma(1-z)$ converges for $\operatorname{Re} z<1$ 
and its singularities are simple poles located at the positive integers with corresponding residues $\frac{(-1)^{n}}{(n-1) !}$. Thus, $\Gamma(z) \Gamma(1-z)$ is a meromorphic function with simple poles at $z=n, n=0, \pm 1, \pm 2, \ldots$ and residues $(-1)^{n}$. But these are precisely the same singularities and residues as are possessed by the function $\pi \operatorname{cosec} \pi z$. This suggests that

$$
\Gamma(z) \Gamma(1-z)=\pi \operatorname{cosec} \pi z
$$

which we confirm as follows.

From (5.2.5) we have, for $0<\operatorname{Re} z<1$,

$$
\Gamma(z) \Gamma(1-z)=\int_{0}^{\infty} e^{-u} u^{z-1} d u \int_{0}^{\infty} e^{-v} v^{-z} d v=4 \int_{0}^{\infty} e^{-x^{2}} x^{2 z-1} d x \int_{0}^{\infty} e^{-y^{2}} y^{-(2 z-1)} d y
$$

where we have introduced the dummy variables of integration $u=x^{2}$ and $v=y^{2}$. Making a further change of integration variables via $r^{2}=x^{2}+y^{2}, \theta=\tan ^{-1}(y / x)$ this becomes

$$
\Gamma(z) \Gamma(1-z)=4 \int_{0}^{\infty} e^{-r^{2}} r d r \int_{0}^{\pi / 2}[\cot \theta]^{2 z-1} d \theta=2 \int_{0}^{\pi / 2}[\cot \theta]^{2 z-1} d \theta, \quad 0<\operatorname{Re} z<1 .
$$

With a final change of variable, $\cot \theta=t$, our product becomes

$$
\Gamma(z) \Gamma(1-z)=2 \int_{0}^{\infty} \frac{t^{2 z-1}}{t^{2}+1} d t=\pi \operatorname{cosec} \pi z, \quad 0<\operatorname{Re} z<1
$$

where the last equality follows from an integral evaluation that we performed in Chapter 3. By analytic continuation, this equality must hold wherever both sides are holomorphic. Thus,

$$
\Gamma(z) \Gamma(1-z)=\pi \operatorname{cosec} \pi z \text { for all finite } z \text {. }
$$

This equation permits easy calculation of $\Gamma(z)$ when $z=\frac{2 n+1}{2}, n=0, \pm 1, \pm 2, \ldots$. Setting $z=\frac{1}{2}$ we have

$$
\left[\Gamma\left(\frac{1}{2}\right)\right]^{2}=\pi \operatorname{cosec} \frac{\pi}{2}=\pi
$$

and hence,

$$
\Gamma\left(\frac{1}{2}\right)=\sqrt{\pi}
$$

Thus, using (5.2.6) , we have

$$
\begin{array}{r}
\Gamma(n+1 / 2)=\frac{(2 n-1)}{2} \frac{(2 n-3)}{2} \cdots \frac{3}{2} \frac{1}{2} \Gamma\left(\frac{1}{2}\right)=\frac{(2 n-1) ! !}{2^{n}} \sqrt{\pi} \\
\Gamma(-n+1 / 2)=\frac{\Gamma\left(\frac{1}{2}\right)}{\frac{(1-2 n)}{2} \frac{(3-2 n)}{2} \ldots \frac{(-3)}{2} \frac{(-1)}{2}}=\frac{2^{n}(-1)^{n} \sqrt{\pi}}{(2 n-1) ! !}
\end{array}
$$


for $n=1,2,3, \ldots$.

Although we now have several ways of analytically continuing our initial integral representation of $\Gamma(z)$, we have yet to find an alternative representation whose domain of validity includes at least part of the left half-plane. A number of possibilities present themselves.

For example, since

$$
\frac{1}{\Gamma(z)}=\frac{\sin \pi z}{\pi} \Gamma(1-z)
$$

is entire, we know from Chapter 3 that it must admit an infinite product representation which is valid everywhere in the finite plane. Indeed, according to equation (3.5.4) we can write

$$
\frac{1}{\Gamma(z+1)}=\frac{1}{\Gamma(1)} e^{c z} \prod_{n=1}^{\infty}\left(1+\frac{z}{n}\right) e^{-z / n}
$$

where $c=-\left.\frac{d}{d z}[\ln \Gamma(z+1)]\right|_{z=0}$ or, since $\Gamma(z+1)=z \Gamma(z)$ and $\Gamma(1)=1$,

$$
\frac{1}{\Gamma(z)}=z e^{c z} \prod_{n=1}^{\infty}\left(1+\frac{z}{n}\right) e^{-z / n} .
$$

To complete the specification of this representation we need only make a more tractable identification of the constant c. Setting $z=1$ in (5.2.13), and using $\Gamma(2)=$ $\Gamma(1)=1$, we find

$$
e^{-c}=\prod_{n=1}^{\infty}\left(1+\frac{1}{n}\right) e^{-1 / n}
$$

or,

$$
c=\sum_{n=1}^{\infty}\left[\frac{1}{n}-\ln \left(1+\frac{1}{n}\right)\right]=\lim _{m \rightarrow \infty}\left[\sum_{n=1}^{m} \frac{1}{n}-\ln m\right] \equiv \gamma
$$

where $\gamma=0.57721566 \ldots$ is a natural number known as the Euler-Mascheroni constant. Thus, we finally obtain the representation

$$
\frac{1}{\Gamma(z)}=z e^{\gamma z} \prod_{n=1}^{\infty}\left(1+\frac{z}{n}\right) e^{-z / n}
$$

for all finite $z$.

This was originally derived by Euler in a somewhat different form. Using (5.2.15) for $\gamma$ we can rewrite (5.2.16) as

$$
\frac{1}{\Gamma(z)}=z \lim _{m \rightarrow \infty} e^{-z \ln m} \prod_{n=1}^{m}\left(1+\frac{z}{n}\right)
$$

and thus obtain

$$
\Gamma(z)=\lim _{m \rightarrow \infty} \frac{m !}{z(z+1) \ldots(z+m)} m^{z}
$$


This is called Euler's formula for $\Gamma(z)$.

Although valid for all finite $z$, (5.2.16) is seldom used other than to calculate the logarithmic derivative of $\Gamma(z)$,

$$
\psi(z) \equiv \frac{d}{d z} \ln \Gamma(z)=-\frac{1}{z}-\gamma+\sum_{n=1}^{\infty}\left[\frac{1}{n}-\frac{1}{z+n}\right] .
$$

The reason for this neglect is evident: neither (5.2.16) nor its alternative form (5.2.17) admits readily to explicit evaluation for specific values of $z$. What we really need, if it exists, is an integral representation of $\Gamma(z)$ that is valid for all finite $z$.

Our starting point in trying to find one is the integral representation that we already possess

$$
\Gamma(z)=\int_{0}^{\infty} e^{-t} t^{z-1} d t, \quad \operatorname{Re} z>0 .
$$

Its integrand has branch points at $t=0$ and $t=\infty$; indeed, it is the behaviour of the integrand in the neighbourhood of $t=0$ that prevents convergence for $\operatorname{Re} z<0$. However, if we join these points by a branch cut running along the positive real axis, we should be able to use the same integrand together with an integration contour that runs just above and below the cut to generate an alternative representation for $\Gamma(z)$. Therefore, let us consider

$$
\int_{C} e^{-t} t^{z-1} d t
$$

where $C$ is the contour shown in Figure 5.8 and $t^{z-1}$ is defined to be the branch

$$
t^{z-1}=e^{(z-1)[\ln |t|+i \arg t]}, \quad 0<\arg t<2 \pi .
$$

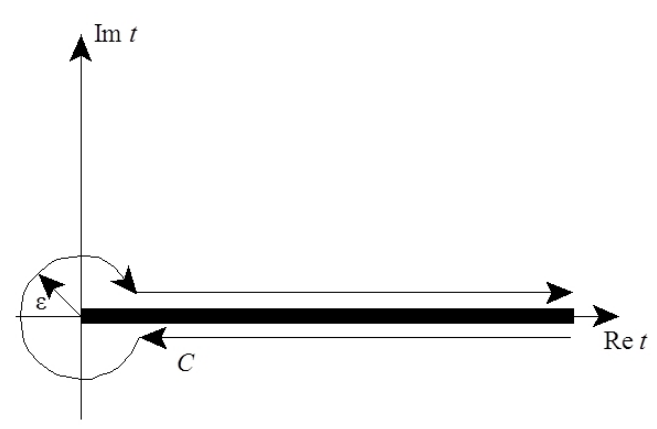

Figure 5.8: The contour in the complex $t$ plane used in the integral (5.2.20). 
With this choice of integration path we not only avoid two troublesome points, we do not cross the cut that joins them. Moreover, at the end-points $t=\infty \pm i \varepsilon$ the integrand vanishes for all finite values of $z$. Thus, our integral converges to a singlevalued function of $z$ for all $z$ in the finite plane.

To find a relationship between the integral and $\Gamma(z)$ we shall temporarily restrict the domain of $z$ to the right half-plane, $\operatorname{Re} z>0$. The contribution of the semi-circular arc about $t=0$ then vanishes in the limit as $\varepsilon \rightarrow 0$ and so,

$$
\int_{C} e^{-t} t^{z-1} d t=\int_{0}^{\infty} e^{-t}\left(e^{2 \pi i} t\right)^{z-1} e^{2 \pi i} d t+\int_{0}^{\infty} e^{-t} t^{z-1} d t=\left(1-e^{2 \pi i z}\right) \int_{0}^{\infty} e^{-t} t^{z-1} d t
$$

or, using (5.2.19),

$$
\int_{C} e^{-t} t^{z-1} d t=2 i \sin \pi z e^{\pi i(z-1)} \Gamma(z) .
$$

Both sides of this equation are entire functions of $z$. Therefore, although derived for $\operatorname{Re} z>0$, it must hold for all finite $z$ and thus provides the representation

$$
\Gamma(z)=\frac{1}{2 i \sin \pi z} \int_{C} e^{-t}(-t)^{z-1} d t, \quad z \neq 0,-1,-2, \ldots
$$

This is sometimes referred to as Hankel's representation. With the help of (5.2.10) we can invert it to obtain an integral representation of $[\Gamma(z)]^{-1}$ that is valid for all finite $z$ without exceptions:

$$
\frac{1}{\Gamma(z)}=\frac{\sin \pi z \Gamma(1-z)}{\pi}=\frac{1}{2 \pi i} \int_{C} e^{-t}(-t)^{-z} d t .
$$

The utility of integral representations stems not only from their large domains of validity but also from the fact that their integration contours can be deformed at will so long as one never passes through singularities of their integrands. Thus, for example, one can often render numerical evaluation very simple by an adroit matching of the contour to the desired value(s) of the function's argument. This will be illustrated in the next Chapter in connection with evaluations for large values of $|z|$. A more immediate demonstration of this versatility is provided by the simple task of evaluating $[\Gamma(z)]^{-1}$ at $z= \pm n, n=0,1,2, \ldots$

When $z$ assumes integer values the integrand in (5.2.22) becomes continuous across the positive real axis and hence, the integration path can be compressed to form a simple closed curve encircling $t=0$. Evaluation of (5.2.22) then involves only a simple application of either the Residue Theorem or Cauchy's Theorem which yield, respectively

$$
\frac{1}{\Gamma(z)}=\left\{\begin{array}{cc}
\frac{1}{(n-1) !} & n>0 \\
0 & n \leq 0
\end{array} .\right.
$$




\subsection{Integral Representations and Integral Transforms}

Evidently, integral representations can play a very significant role in the definition and continuation of analytic functions. We shall conclude this Chapter with some general comments about them. And to start with, we shall determine the circumstances under which an integral representation definition is valid.

Theorem: Let $G(z, t)$ be a continuous function of both variables when $z$ lies in a simply connected domain $D$ and $t$ lies on a simple curve $C$. Further, for each such value of $t$, let $G(z, t)$ be holomorphic within $D$. Then, the function

$$
f(z)=\int_{C} G(z, t) d t
$$

is also holomorphic within $D$ and its derivatives of all orders may be found by differentiating under the integration sign, provided that

1. $C$ is of finite length, or

2. if $C$ is of infinite length, the integral is uniformly convergent for $z$ contained in any closed region interior to $D$.

Proof: Let $\Gamma$ denote any simple closed curve in $D$. Then,

$$
\int_{\Gamma} f(z) d z=\int_{\Gamma}\left\{\int_{C} G(z, t) d t\right\} d z=\int_{C}\left\{\int_{\Gamma} G(z, t) d z\right\} d t=0
$$

and hence, by Morera's Theorem, $f(z)$ is holomorphic in $D$. Here we have used the fact that the order of integration of an iterated integral with a continuous integrand can always be interchanged if it is uniformly convergent or finite. We have also used the holomorphy of $G(z, t)$ in $D$ and Cauchy's Theorem to obtain the final equality with zero.

Similarly, using Cauchy's Differentiation Formula we have

$$
\frac{d f(z)}{d z}=\frac{1}{2 \pi i} \int_{\Gamma} \frac{f(\zeta)}{(\zeta-z)^{2}} d \zeta=\int_{C}\left\{\frac{1}{2 \pi i} \int_{\Gamma} \frac{G(\zeta, t)}{(\zeta-z)^{2}} d \zeta\right\} d t=\int_{C} \frac{\partial G(z, t)}{\partial z} d t
$$

The most frequently encountered integral representations are of the somewhat specialized form known as integral transforms,

$$
f(z)=\int_{C} K(z, t) g(t) d t .
$$

The function $K(z, t)$ is called the kernel of the transform while $g(t)$ is known as the spectral function. Each distinct type of transform corresponds to a specific choice of kernel and of integration contour $C$ and each has an "existence theorem" that determines the class of spectral functions $g(t)$ and the domain of $z$ for which the integral converges. 
We were introduced to the Hilbert transform,

$$
f(z)=\frac{1}{\pi} \wp \int_{-\infty}^{\infty} \frac{g(t)}{t-z} d t,
$$

in the course of our discussion of dispersion relations in Section 4.1. Other transforms that we will have occasion to discuss in subsequent Chapters include the Fourier transform,

$$
f(z)=\frac{1}{2 \pi} \int_{-\infty}^{\infty} e^{i t z} g(t) d t,
$$

and its two close relatives, the Laplace transform

$$
f(z)=\frac{1}{2 \pi i} \int_{c-i \infty}^{c+i \infty} e^{t z} g(t) d t
$$

and the Mellin transform

$$
f(z)=\frac{1}{2 \pi i} \int_{c-i \infty}^{c+i \infty} z^{t} g(t) d t .
$$

As we shall see, transforms arise naturally in the solution of boundary value problems where the form of the differential equation together with the nature of the boundary conditions determines more or less uniquely which type of transform to use. 